\title{
Erratum to: Transcriptionally and Phylogenetically Analyzing the P Protein Gene of Glycine Decarboxylase for Understanding the Evolution of $\mathrm{C}_{3}-\mathrm{C}_{4}$ Species in Brassicaceae
}

\author{
Chunyu Zhang ${ }^{1}$, In-ae Park ${ }^{2}$, Fangsen $\mathrm{Xu}^{1}$, Maoteng $\mathrm{Li}^{3}$, Yong Pyo $\mathrm{Lim}^{{ }^{2}}$, and Jinling Meng ${ }^{1}$ \\ ${ }^{1}$ National Key Laboratory of Crop Genetic Improvement, Huazhong Agricultural University, Wuhan 430070, China \\ ${ }^{2}$ Department of Horticulture, Chungnam National University, Daejeon 305-764, Korea \\ ${ }^{3}$ College of Life Science and Technology, Huazhong University of Science and Technology, Wuhan 430074, China \\ *Corresponding author: yplim@cnu.ac.kr
}

(C) Korean Society for Horticultural Science and Springer 2013

Erratum to: Hort. Environ. Biotechnol. 52(4):427-434. 2011.

DOI 10.1007/s13580-011-0215-4

The original version of this article contained an error in the Fig. 2C.

The correct Fig. 2 is reproduced below.

A
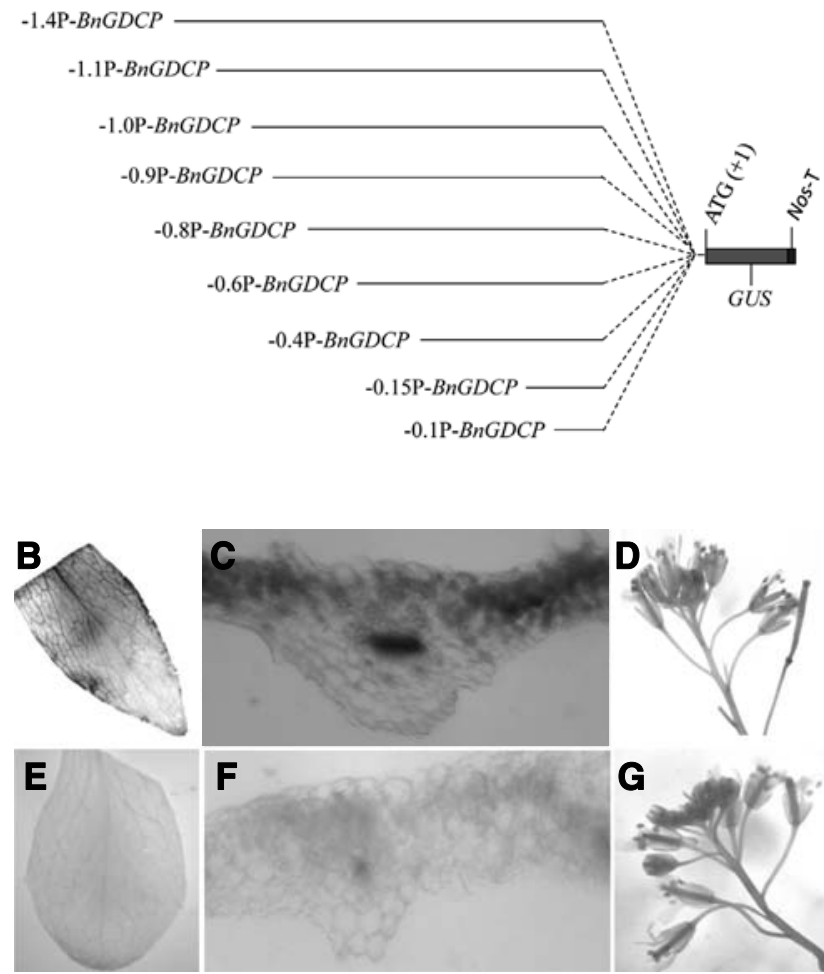

G

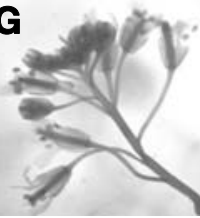

Fig. 2. Deletion analysis of the promoter of $B n G D C P$ in $C_{3}$ species (Arabidopsis). (A) Structures of the deleted promoter/GUS chimerical genes used for Arabidopsis transformation. $-n P-B n G D C P$ represents a series of deleted promoters located at "n" position upstream of AUG initiation codon of BnGDCP. (B), (C), and (D) show the histochemical localization of GUS activity in a whole leaf, leaf section, and floral tissues of Arabidopsis plant transformed with $-0.9 \mathrm{P}-B n G D C P$ :GUS construct, respectively. $(E),(F)$, and $(G)$ show the histochemical localization of GUS activity in a whole leaf, leaf section, and floral tissues of Arabidopsis plants transformed with $-0.8 \mathrm{P}-B n G D C P: G U S$ construct, respectively.

The online version of the original article can be found under 